\title{
A profile of emergency readmissions to Department of Surgery, Victoria Hospital, Blackpool
}

\author{
G Samra*, K Mehta \\ From International Conference for Healthcare and Medical Students 2011 \\ Dublin, Ireland. 4-5 November 2011
}

\section{Introduction}

For several years an increasing number of patients are being readmitted to hospital as an emergency soon after their initial discharge. The reasons behind such readmissions are highly complex, and studies have so far failed to identify the definitive drivers of this trend.

\section{Aims}

To create a profile of emergency readmissions to the Department of Surgery, Victoria Hospital, Blackpool (June-July 2010) encompassing the following profile elements: Patient demographics, source of admission, surgical specialty involved in patient care, duration of hospital stay during admissions, gap between discharge and readmission, reason for readmission, surgical procedure and investigation during admissions.

\section{Methods}

Data Source: Informatics Department and patient admission register on Surgical Assessment Unit (Retrospective data collection); Data Compilation: Patient Admission Database (PAD); $\mathrm{N}=68$.

\section{Results}

Age distribution: $33.8 \%$ of patients readmitted were $41-60$ years old and $29.4 \%$ were $>61$ years old. Gender distribution: No significant difference in the gender distribution of patients readmitted. Source of first admission: $61.7 \%$ of patients readmitted were referred from GP and $33.8 \%$ of patients readmitted were referred from $A E$ as the first source of admission. Specialty distribution: General Surgery 77\%; Hepato-billiary 8\%; Breast 4\%; Vascular 4\%; Colo-rectal 3\%; Urology 4\%. Duration of stay: The average duration of stay during the first admission was 5 days. The average gap between discharge and readmission was 7 days. The average duration of stay during the first admission was 10 days. Reason for readmission: Same Diagnosis 57\%; Pain 18\%; Bleeding 4\%; Infection 6\%; Constipation 4\%; Nausea-vomiting 2\%; Other reason 9\%. Procedure on first admission (top three): 1. Appendecectomy, 2. Incision and drainage of abcess, 3. Circumscision. Procedure on second admission (top 3) 1. Catheter change, 2. Incision and drainage of abcess, 3. Cholecystectomy.

\section{Conclusions}

Elderly patients admitted for less than a week from primary care as emergency admissions with general surgical complaints have a high risk of readmission. This study identifies patient, health-care service and disease variables that relate to high readmission rates and lays the foundation for addressing pertinent issues.

Published: 9 July 2012

\section{doi:10.1186/1753-6561-6-S4-P2}

Cite this article as: Samra and Mehta: A profile of emergency readmissions to Department of Surgery, Victoria Hospital, Blackpool. BMC Proceedings 2012 6(Suppl 4):P2.

Blackpool Fylde and Wyre NHS Hospitals Trust, UK

(c) 2012 Samra and Mehta; licensee BioMed Central Ltd. This is an Open Access article distributed under the terms of the Creative 